\title{
Vivências de sofrimento e prazer das acadêmicas de enfermagem nas maternidades
}

\author{
Suffering and pleasure experiences of nursing undergraduate students in maternity \\ Vivencias de sufrimiento y placer de estudiantes de enfermeríaen las maternidades
}

\author{
Jane Márcia Progianti'; Marina Nunes de Souzal'; Elias Barbosa de Oliveira"li; \\ Fernanda Alves Bittencourt Rodrigues ${ }^{\prime}$; Juliana Amaral Pratav, Octavio Muniz da Costa Vargens ${ }^{v /}$
}

\begin{abstract}
RESUMO
Objetivo: identificar as vivências de sofrimento e de prazer dos acadêmicos de enfermagem frente à organização do trabalho das enfermeiras obstétricas na maternidade. Método: estudo exploratório e qualitativo, com 13 acadêmicas de enfermagem de uma instituição do ensino superior privada do Rio de Janeiro. Dados coletados em abril e maio de 2018, por meio de entrevistas semiestruturadas, submetidos à análise de conteúdo e discutidos à luz do referencial teórico de Christopher Dejours. Resultados: durante o estágio supervisionado, as vivências de sofrimento associaram-se à percepção de falta de reconhecimento e à identificação da violência obstétrica. As vivências de prazer foram relacionadas ao reconhecimento das mulheres pelo cuidado prestado. Conclusão: são necessárias estratégias pedagógicas aplicadas nos cenários de prática, capazes de problematizar o contexto laboral e suas implicações sobre o trabalhador, com o intuito de constituir enfermeiras com atitudes transformadoras da realidade e dispostas a lutar pela valorização e pelo reconhecimento da enfermagem obstétrica. Descritores: Trabalho; saúde do trabalhador; trabalho feminino; enfermagem.
\end{abstract}

\section{ABSTRACT}

Objective: to identify experiences of nursing students on suffering and pleasure in relation to the work organization of obstetric nurses in the maternity ward. Method: exploratory and qualitative study, with 13 nursing undergraduate students from a private institution in Rio de Janeiro, Brazil. Data were collected in April and May 2018 through semi-structured interviews. Content analysis and Christopher Dejours' theoretical framework was used to data analysis. Results: during the supervised internship, the experiences of suffering were associated with the perception that the obstetric violence is not recognized nor identified. The experiences of pleasure are related to the women's recognition of the care provided. Conclusion: it is necessary to adopt pedagogical strategies to be possible to problematize the work context as well its repercussions on the worker, in order to prepare nurses with reality-transforming attitudes and dispositions to fight for the appreciation and recognition of obstetric nursing.

Descriptors: Work; occupational health; women, work; nursing.

\section{RESUMEN}

Objetivo: identificar experiencias de estudiantes de enfermería sobre sufrimiento y placer en relación con la organización del trabajo de las enfermeras obstétricas en el sector de la maternidad. Método: estudio exploratorio y cualitativo, con 13 estudiantes de enfermería de una institución privada de educación superior en Río de Janeiro, Brasil. Los datos se recopilaron en abril y mayo de 2018 a través de entrevistas semiestructuradas. El análisis de contenido y el marco teórico de Christopher Dejours se utilizaron para analizar los datos. Resultados: durante el entrenamiento supervisado, las experiencias de sufrimiento se asociaron con la percepción de que la violencia obstétrica no se reconoce ni se identifica. Las experiencias de placer están relacionadas con el reconocimiento de las mujeres de la atención brindada. Conclusión: es necesario adoptar estrategias pedagógicas para poder problematizar el contexto laboral y sus repercusiones en el trabajador, a fin de preparar a las enfermeras con actitudes y disposiciones transformadoras de la realidad para luchar por la apreciación y el reconocimiento de la enfermería obstétrica.

Descriptores: Trabajo; salud del trabajador; mujeres trabajadoras; enfermería.

\section{INTRODUÇÃO}

No âmbito do Sistema Único de Saúde (SUS), o avanço das reformas neoliberais no Brasil foi impactante tendo em vista que a redução do papel do Estado determinou restrições no financiamento, culminando no sucateamento das instituições públicas de saúde acompanhado do surgimento de modelos flexíveis de gestão dos serviços e dos recursos humanos ${ }^{1,2}$.

'Enfermeira. Doutora em Enfermagem. Professora Associada. Universidade do Estado do Rio de Janeiro. Brasil. E-mail: jmprogi@uol.com.br

"Enfermeira. Especialista. Acadêmica do curso de mestrado em Enfermagem. Universidade do Estado do Rio de Janeiro. Brasil. E-mail: ninhamarina@oi.com.br I"Enfermeiro. Doutor em Enfermagem. Professor Associado. Universidade do Estado do Rio de Janeiro. Brasil. E-mail: eliasbo@oi.com.br

IVEnfermeira. Especialista. Acadêmica do curso de mestrado em Enfermagem. Bolsista da Coordenação de Aperfeiçoamento Pessoal de Nível Superior (CAPES). Universidade do Estado do Rio de Janeiro. Brasil.E-mail: fernanda.abr@hotmail.com

voutora em Enfermagem. Professora Assistente. Universidade do Estado do Rio de Janeiro. Brasil. E-mail: juaprata@gmail.com

viDoutor em Enfermagem. Professor Titular. Universidade do Estado do Rio de Janeiro. Brasil. E-mail: omcvargens@uol.com.br 
Sob essa lógica organizacional, verifica-se a expansão da rede assistencial, principalmente por meio de Organizações Sociais, e a coexistência de diferentes modalidades de vinculação trabalhista. Esta atual conformação do campo laboral associa-se com a precarização do trabalho no SUS, expressa na multiplicidade de vínculos dos trabalhadores com a administração pública, na instabilidade de algumas formas de relação laboral e na desregulação das condições de trabalho, que retira a autonomia dos trabalhadores e os coloca em situações de vulnerabilidade, no sentido da insegurança, intensificação e desmotivação².

Como exemplos de flexibilidade no setor público, destacam-se a Estratégia Saúde da Família e, mais recentemente, a Rede Cegonha e o Programa Cegonha Carioca. Estas últimas consistem em iniciativas governamentais para melhorar os indicadores de saúde materna e neonatal a partir da implementação de uma rede de cuidados qualificados e seguros, condizente com o modelo assistencial humanizado ${ }^{3,4}$.

Nesse contexto, o mercado laboral do SUS absorveu um grande quantitativo de enfermeiras obstétricas, contudo, constata-se que a organização do trabalho dessas especialistas nas maternidades se caracteriza pela flexibilização das relações trabalhistas e exigência por produtividade, conformando um processo de trabalho multifuncional, polivalente, com tarefas hierarquizadas e realizadas em ritmo intenso que desvelam a intensificação ${ }^{4-6}$. Além disso, acrescentam-se os baixos salários, a divisão sexual e a convivência conflituosa entre os diferentes profissionais que atuam na assistência ao parto, enquanto condições que revelam a desvalorização do trabalho da enfermagem obstétrica nas maternidades e contribuem para a desmotivação das enfermeiras ${ }^{5,7-9}$.

Para além dos efeitos deste panorama sobre a especialidade, é importante ponderar que o espaço sócioprofissional da maternidade é também um campo de formação prática, onde os acadêmicos de enfermagem se inserem durante o estágio curricular supervisionado. Esta vivência é de extrema relevância para o processo formativo, entretanto, o contexto atual do mundo do trabalho impõe desafios, pois as relações profissionais conflituosas, os dilemas éticos e a falta de reconhecimento são questões da organização do trabalho das enfermeiras que interagem com as subjetividades dos discentes, conformando vivências diversificadas ${ }^{10,11}$.

Frente ao exposto, esse estudo objetivou identificar as vivências de sofrimento e de prazer dos acadêmicos de enfermagem frente à organização do trabalho das enfermeiras obstétricas no âmbito das maternidades. Sob a ótica de futuros profissionais, esta pesquisa é relevante para a reflexão acerca de novas estratégias de ensino e aprendizagem aplicadas à formação prática em enfermagem, com vistas à problematização da influência da organização do trabalho sobre a produção de vivências de prazer, sofrimento, desgaste e satisfação dos trabalhadores, enquanto um recurso necessário para a compreensão da realidade laboral e desenvolvimentos de atitudes transformadoras.

\section{REFERENCIAL TEÓRICO}

Este estudo está ancorado na Psicodinâmica do Trabalho que possui as seguintes categorias teóricas de análise: a organização do trabalho, composta pela organização do contexto do trabalho; as condições e relações laborais; a mobilização subjetiva do trabalhador, com vivências de prazer/sofrimento, e as estratégias de enfrentamento adotadas diante das adversidades ${ }^{12}$.

Nessa abordagem teórica, a organização do trabalho, que compreende a divisão do trabalho (divisão e conteúdo das tarefas, cadência e tempo) e divisão dos indivíduos (relações de poder, responsabilidade, hierarquia, comando e controle), prescreve atividades e relações humanas para o trabalhador. Desse modo, é um conceito que se apropria de duas ideias de trabalho: o prescrito, definido, como a atividade preestabelecida; e o real, o que é realizado concretamente ${ }^{12,13}$.

A relação entre o trabalho prescrito, real e as subjetividades do trabalhador é uma construção dialética na qual a organização do trabalho pode ser fonte de sofrimento e prazer. Nesse sentido, o sofrimento no trabalho surge quando o trabalhador percebe que a relação entre si mesmo e a organização do trabalho encontra-se bloqueada, isto é, quando a mesma não permite a subversão do trabalho prescrito por meio do uso da inteligência prática ou da criatividade. Já o prazer, advém de uma forma de organização que permite transgressão do trabalho prescrito, estruturação psíquica e expressão da identidade do trabalhador ${ }^{12,14,15}$

\section{METOdologia}

Trata-se de uma pesquisa exploratória, com abordagem qualitativa, realizada com 13 acadêmicos de enfermagem de uma instituição do ensino superior (IES) privada do Rio de Janeiro, sendo onze mulheres e dois homens.

Como critérios de inclusão, definiu-se: a participação voluntária; ter matrícula ativa e regular; e ter aprovação na disciplina "estágio supervisionado em saúde da mulher", do oitavo período da graduação. A adoção deste último justifica-se, pois a referida disciplina oportuniza o desenvolvimento de atividades práticas e o contato com a realidade da atuação profissional das enfermeiras obstétricas na maternidade. Como critérios de exclusão, considerou-se apresentar frequência inferior a $80 \%$ da carga horária total da disciplina. 
A captação das participantes aconteceu a partir de um levantamento nominal dos acadêmicos aprovados na disciplina, obtido pela autora que realizou a coleta dos dados, que era docente do quinto período da graduação e, na mesma época, mestranda. Frente ao total de 40 alunos, adotou-se a técnica da "bola de neve", selecionando, aleatoriamente, a "semente" para que esta indicasse participantes potenciais ${ }^{16}$. Assim, realizou-se um contato prévio com cada indicado, seguido do convite para a participação, alcançando 13 acadêmicos que se configuraram como participantes do estudo, sem perdas.

A coleta de dados aconteceu nos meses de abril e maio de 2018, por meio de entrevistas semiestruturadas, seguindo um roteiro dividido em duas partes, sendo a primeira destinada à apreensão de características gerais dos participantes e a segunda contendo uma questão norteadora: "Fale-me sobre as situações do trabalho da enfermeira obstétrica na maternidade que lhe causaram sofrimento e prazer durante o estágio". Cabe ressaltar que foi realizado um teste piloto e que não houve a necessidade de alterações no roteiro.

As entrevistas foram realizadas individualmente por uma das autoras do estudo, em local reservado e escolhido pelo participante, gravadas em arquivos de áudio e transcritas logo em seguida, o que permitiu vislumbrar o momento em que os discursos se tornaram redundantes, indicando a saturação dos dados e determinando o encerramento da coleta de dados ${ }^{17}$.

Os dados foram examinados pela análise de conteúdo de Bardin ${ }^{18}$ e discutidos à luz do referencial teórico de Cristophe Dejours. Da pré análise, seguiu-se para exploração do material, o qual foi recortado em unidades de registro, as quais foram organizadas em unidades de significação e reagrupadas, progressivamente, até as duas categorias analíticas finais: "Vivências de sofrimento", com duas subcategorias, e "Vivências de prazer".

Atendendo à Resolução no 466/2012 ${ }^{19}$, os participantes assinaram um Termo de Consentimento Livre e Esclarecido, explicitando a participação voluntária e a manutenção do anonimato. Para tanto, adotou-se a letra $\mathrm{E}$, concernente ao termo entrevistada, seguida de um algarismo arábico, representando a ordem de realização das entrevistas.

A pesquisa foi submetida ao Comitê de Ética em Pesquisa do Hospital Universitário Pedro Ernesto da Universidade do Estado do Rio de Janeiro, sob o parecer de aprovação CAAE 82003517.6.0000.5259, de março de 2018.

\section{RESULTADOS E DISCUSSÃO}

\section{Vivências de sofrimento}

Nesta categoria, as falas dos acadêmicos de enfermagem acerca do trabalho das enfermeiras obstétricas nas maternidades confluíram para vivências de sofrimento associadas à percepção de falta de reconhecimento e à identificação de situações da violência obstétrica.

\section{Percebendo a falta de reconhecimento do trabalho das enfermeiras obstétricas}

Para os participantes deste estudo, a organização do trabalho das enfermeiras obstétricas na maternidade produziu representações negativas expressas na falta de reconhecimento profissional:

Acho que o enfermeiro não tem o reconhecimento que deveria, porque a gente estuda... Não para só na graduação![...] Temos muita coisa para oferecer e, muitas vezes, não somos reconhecidos! (E4).

As pessoas não conhecem o trabalho e não valorizam! Porque ainda tem muito a figura do médico como aquele que faz o parto! Então, ainda não está na cabeça das pessoas que o enfermeiro tem total capacidade para isso! (E7).

Acho o trabalho importante, mas reconhecido não! É uma luta a enfermagem "fazer" um parto! Não é reconhecido pelo sistema, pela categoria médica, instituição, população... (E8).

Uma rotina pesada, cansativa e com baixos salários! A falta de boas condições de trabalho, como falta de profissionais e insumos, pois se você possui ao seu alcance boas condições de trabalho, seu desempenho acaba sendo mais efetivo, mais favorável, do contrário você acaba ficando limitado (E12).

As falas revelam que a percepção de desvalorização e de falta de reconhecimento social da profissão geraram sofrimento nos acadêmicos de enfermagem durante o estágio supervisionado. Este período da formação é de extrema importância, visto que a inserção dos discentes nos serviços do SUS e a vivência do exercício profissional na organização do trabalho em saúde oportuniza a aplicação de conhecimentos, a incorporação de atitudes, o desenvolvimento de habilidades, criatividade e autonomia com vistas à consolidação de competências específicas da enfermagem ${ }^{10,11,20}$.

A partir desta aproximação com o mundo do trabalho, o discente articula teoria e prática, adquire experiências significativas, problematiza e reflete criticamente sobre o saber-fazer profissional, adquire disposições para transformar a realidade e constrói referentes identitários sobre o ser enfermeira ${ }^{10-11,20}$. 
Porém, ao deparar-se com conflitos e situações de cuidado e de relações interpessoais moralmente inadequadas que expressam desrespeito, os discentes se sentem angustiados, desencorajados e impotentes, percebendo-se na vivência de sofrimento ${ }^{21-23}$.

Neste sentido, acrescenta-se que a desvalorização do trabalho da enfermagem captada pelos participantes do estudo tem origem no processo histórico de constituição da profissão, essencialmente feminina e associada à caridade, abnegação e obediência. Ainda, a persistência do modelo assistencial tecnocrático em alguns cenários de atenção à saúde contribui para reforçar o saber médico em detrimento à ciência do cuidado de enfermagem, reproduzindo a ideia equivocada de dedicação vocacional da enfermeira e colocando a profissão numa posição de menor prestígio e de invisibilidade social, principalmente na organização do trabalho hospitalar ${ }^{24,25}$.

Estas questões tornam-se ainda mais visíveis no âmbito da assistência ao parto, pois nas maternidades, apesar da atuação das enfermeiras obstétricas ser legitimada pelas políticas públicas de saúde da mulher e amparada por dispositivos legais, a hierarquia e a divisão sexual existentes no campo obstétrico hospitalar impõem à essas especialistas relações conflituosas que, somadas às condições extenuantes de trabalho nestes locais, desvelam a desvalorização do trabalho feminino ${ }^{5,25}$.

Corroborando, destaca-se a histórica divisão técnica e social do trabalho da enfermagem, que dificultam a delimitação das atribuições e dos papéis de cada um dos componentes desta equipe e colaboram para a construção de imagens e representações sociais distorcidas acerca do ser enfermeira e de suas especialidades, enquanto profissional desvalorizado, com baixa remuneração e subordinado, especialmente, à medicina ${ }^{24-26}$.

$\mathrm{Na}$ atualidade, o desprestígio, a desvalorização, a invisibilidade e a falta de reconhecimento social são significados atribuídos ao contexto do trabalho das enfermeiras obstétricas nas maternidades, os quais são percebidos pelos acadêmicos de enfermagem e constituem vivências de sofrimento. Durante a formação, estas percepções podem repercutir negativamente sobre o perfil da futura enfermeira, pois influencia no processo de construção da identidade profissional e gera desmotivação para a profissão. Por outro lado, ao se inserirem no mundo laboral, essa conjuntura se interrelaciona com as subjetividades dos trabalhadores e pode conduzir, à longo prazo, à desmobilização e ao sofrimento patogênico no trabalho ${ }^{14}$.

\section{Identificando situações de violência obstétrica na maternidade}

Segundo os acadêmicos de enfermagem, a identificação de situações de violência obstétrica também emergiu como uma vivência de sofrimento durante o estágio supervisionado na maternidade:

Presenciei várias formas de violência obstétrica que ia desde a repreensão do profissional para fazer com que a paciente parasse de gritar durante o trabalho de parto (E2).

A forma como a médica falou... A impaciência porque o parto em si demora! O momento que era para ser prazeroso, acaba se tornando doloroso. É marcante! Senti pelo que a paciente ouviu... Aquelas agressões verbais são muito ruins! Me marcou (E6).

Obrigar a paciente a ficar em uma determinada posição, pegar as perneiras e obrigar a paciente a ficar lá... Foram ações realizadas pela médica obstétrica e não podemos falar nada! (E13).

Nota-se que as formas de violências obstétricas mais expressivas nas falas dos acadêmicos de enfermagem foram aquelas exercidas por médicos através da violência física, expressa no desrespeito ao direito à livre movimentação e à escolha da posição no momento do parto, e da violência psicológica, revelada por meio de abuso verbal, tratamento rude, gritos, repreensão, humilhação e intimidação.

A expressão violência obstétrica compreende manifestações de desrespeito aos direitos humanos das mulheres durante a assistência ao parto ou no aborto. Sob esta ótica, inclui qualquer ato exercido por profissionais da saúde que configure: atenção desumana; omissão ou negligência de cuidados; discriminação social; maus-tratos físico, psicológico e/ou verbal; realização de procedimentos desnecessários, prejudiciais e sem o consentimento prévio da mulher; falta de confidencialidade; desrespeito ao direito ao acompanhante, à informação e à privacidade ${ }^{27-29}$.

Considerando que todas as mulheres têm direito ao acesso aos serviços de saúde e às informações, assim como à participar das decisões sobre seu corpo, incluindo uma assistência digna, de qualidade, segura e respeitosa, a violência obstétrica é uma violação dos direitos humanos fundamentais, pois, independente do modo como se manifeste, ela representa uma ameaça à vida, à saúde, à integridade física e psíquica da mulher ${ }^{30}$.

Cabe ressaltar, que esta forma de violência se relaciona com a modalidade de assistência típica do modelo biomédico, marcado pela hierarquia institucional que se revela na transformação do parto em um evento patológico, onde a mulher é um objeto de intervenção, destituída de autonomia para escolher e decidir sobre seu próprio corpo diante do poder médico socialmente cristalizado ${ }^{27,31}$. 
As atitudes e condutas geradas a partir desta visão de mundo medicalizada podem ser vislumbradas sob a perspectiva de gênero, pois os profissionais médicos, detentores de saberes técnico-científicos e respaldados em relações desiguais de poder, usam de sua autoridade naturalizada na sociedade androcêntrica para se apropriar do corpo feminino 27 .

Neste cenário, a organização do trabalho na maternidade se caracteriza por desigualdades de gênero, pois saberes associados ao feminino são vistos como hierarquicamente inferiores àqueles de origem masculina, o que transparece na dominação do feminino pelo masculino e nas relações de poder existentes entre medicina e enfermagem. Assim, o processo de trabalho da enfermagem, agrega hierarquização, sobrecarga, esgotamento, desvalorização e precarização uma vez que se trata de uma profissão, predominantemente, feminina, com atributos associados ao cuidado, paciência e zelo $6,8,25,26,32$.

Inseridos neste contexto durante a formação, os acadêmicos de enfermagem percebem a desvalorização do trabalho da enfermeira e identificam práticas assistenciais condizentes com situações de violência obstétrica, as quais foram relacionadas, na maioria das vezes, com comportamentos apresentados por outros profissionais. Essas percepções se chocam com as disposições internalizadas pelos discentes durante a graduação, configurando vivências de sofrimento mental ${ }^{10,11}$.

Sob a ótica da psicodinâmica do trabalho, tal constatação remete à ideia de trabalho prescrito, que corresponde aos saberes teóricos e práticos adquiridos no campo do ensino, e o trabalho real, enquanto conhecimentos e práticas adaptados à realidade da organização do trabalho na maternidade. Este distanciamento entre o ensino e os serviços de saúde engendra a desarticulação teórico-prática que, ao ser percebida pelos discentes durante o estágio curricular supervisionado, produz representações negativas e gera sofrimento, pois este sentimento surge do descompasso entre as subjetividades do indivíduo e as condições percebidas da organização do trabalho, ou seja, diante da divergência entre trabalho prescrito e real ${ }^{33}$.

\section{Vivência de Prazer}

Nesta categoria, os discursos dos acadêmicos de enfermagem que desvelaram vivências de prazer não foram gerados por percepções positivas acerca da organização do trabalho das enfermeiras obstétricas na maternidade, mas se mostraram ancorados no reconhecimento das atividades desenvolvidas pelos discentes durante o estágio curricular supervisionado, por parte das mulheres e de seus familiares:

Em geral, o que dá prazer é o olhar de agradecimento do cliente e da família! E a fala gentil pelo acolhimento recebido pela enfermagem e entre outros profissionais (E1).

Quando alguém reconhece o esforço que a gente tem! Quando a gente é reconhecida... Já sou quase uma enfermeira! [...] Você se sente muito mais útil pra sociedade quando consegue entender bem o que está fazendo! Acho que isso traz prazer! Ficamos felizes (E4).

Os agradecimentos e reconhecimento do serviço realizado (E5).

$O$ reconhecimento e acolhimento do paciente! (E12).

As falas revelam que, ao se inserirem na organização e no processo de trabalho das enfermeiras na maternidade, a vivência de prazer dos acadêmicos de enfermagem adveio, somente, do julgamento da utilidade do cuidado percebido como reconhecimento e valorização do trabalho desenvolvido, com repercussões sobre a motivação e satisfação dos mesmos com a futura profissão.

O reconhecimento compreende uma recompensa moral e simbólica, fruto da contribuição do indivíduo na dinamicidade da organização do trabalho, por meio do esforço, da persistência, da resistência ao fracasso, do engajamento da subjetividade e da inteligência mobilizada para a solução de problemas laborais $\operatorname{cotidianos}^{34}$.

Para a Psicodinâmica do Trabalho, o trabalhador desenvolve suas atividades orientado pela relação, contribuição e retribuição. Nessa tríade, o reconhecimento é uma forma de retribuição simbólica advinda de sua contribuição. Entretanto, esta retribuição não é automática, mas consequente aos significados e valores associados ao trabalho, de modo que o reconhecimento pode ser atribuído pela chefia, subordinados ou pacientes, exaltando a importância do trabalhador na esfera laboral, e/ou conferido pelos pares, gerando no indivíduo o sentimento de pertencimento à um grupo profissional ${ }^{13}$

Por outro lado, o não reconhecimento denota a invisibilidade do trabalhador na organização do trabalho, desencadeando sofrimento por ausência de significação social pelas atividades laborais, com potencial para comprometer a saúde física e mental por desestabilização do referencial que sustenta sua identidade profissional ${ }^{12,13}$.

Assim, a percepção de reconhecimento é decisiva no mecanismo de mobilização subjetiva, enquanto processo por meio do qual o trabalhador utiliza seus recursos intelectuais e sua subjetividade para transformar as situações geradoras 
de sofrimento em fontes de prazer, permitindo o resgate do sentido do trabalho e, consequentemente, da realização profissional ${ }^{35}$.

Para os participantes deste estudo, as demonstrações de gratidão, expressas em gestos, atitudes e comentários positivos, funcionaram como recursos que mobilizaram suas subjetividades, pois, ao perceberem o reconhecimento pelo cuidado, foi possível ressignificar as vivências de sofrimento e alcançar o prazer durante o estágio supervisionado ${ }^{26}$.

\section{CONCLUSÃO}

Este estudo evidenciou que a organização do trabalho das enfermeiras obstétricas na maternidade produziu percepções negativas para os acadêmicos de enfermagem, expressas na falta de reconhecimento e na identificação de situações da violência obstétrica, as quais se configuraram como vivências de sofrimento durante o estágio curricular supervisionado. Por outro lado, as vivências de prazer foram geradas a partir do reconhecimento por parte das mulheres e de seus familiares pelo cuidado realizado.

Cabe ponderar que estas constatações acerca da experiência de aproximação dos discentes com o mundo do trabalho das enfermeiras podem impactar na constituição do perfil da futura enfermeira e no processo de construção da identidade profissional, com potencial para a desmotivação dos estudantes para a profissão. Neste sentido, os resultados desta pesquisa revelam a fragilidade existente na formação no tocante à articulação do campo do ensino com o campo laboral e a importância de promover um ensino prático, crítico e contextualizado com a realidade dos serviços de saúde, com vistas à amenizar o conflito entre trabalho prescrito, retratado pelo ensino teórico-prático, e o trabalho real, exposto como o ensino prático nas condições oferecidas pela organização do trabalho.

Ainda, o fato dos discentes apreenderem o reconhecimento no trabalho, somente, por meio de demonstrações de agradecimento manifestadas pelas mulheres sugere a invisibilidade do trabalho da enfermeira para os agentes que ocupam melhores posições na hierarquia do campo obstétrico. Este achado engendra importantes reflexões, pois, ainda que haja reconhecimento pelo cuidado, este se dá por atributos femininos associados ao amor, paciência e zelo, os quais reproduzem estereótipos históricos da profissão e não favorecem a valorização do trabalho da enfermeira no atual contexto laboral, desconsiderando o arcabouço teórico-científico que determina as distinções presentes nas práticas assistenciais, gerenciais, políticas e sociais da enfermagem, sobretudo da enfermagem obstétrica.

Como limitação da pesquisa que impede generalizações, destaca-se o fato de se tratar de um estudo local realizado em uma única IES privada, o que aponta para a necessidade de novas investigações de maior abrangência, abordando também o ensino da graduação de enfermagem em instituições públicas. No entanto, seus resultados são válidos, pois evidenciam a imprescindibilidade de estratégias pedagógicas aplicadas nos cenários de formação prática, capazes de problematizar o contexto laboral no qual os acadêmicos se inserem e suas implicações sobre as vivências do trabalhador, com o intuito de constituir futuras enfermeiras dotadas de atitudes transformadoras da realidade e com disposições para lutar pela valorização e pelo reconhecimento da enfermagem obstétrica no mundo do trabalho.

\section{REFERÊNCIAS}

1. Marques APP. Productive restructuring and the reallocation of work and employment. A survey of the "new" forms of social inequality. Ciênc saúde coletiva [Internet], 2013 [cited 2018 aug 23]; 18(6): 1545-54. DOI: http://dx.doi.org/10.1590/S141381232013000600007.

2. Martins MIC, Molinaro A. Productive restructuring and its impact on labor relations in the public health services in Brazil. Ciênc saúde coletiva [Internet], 2013 [cited 2018 aug 23]; 18(6): 1667-76. DOI: http://dx.doi.org/10.1590/S141381232013000600018

3. Pereira ALF, Nicácio MC. Formation and professional insertion of obstetrical nursing residency graduates. Rev. enferm. UERJ [Internet], 2014 [cited 2018 aug 23]; 22(1): 50-6. Available from: https://www.epublicacoes.uerj.br/index.php/enfermagemuerj/article/view/11418

4. Progianti JM, Prata JA, Barbosa PM. Healthcare and productive restructuring: effects of increased flexibility on maternity hospitals in the Cegonha Carioca program. Rev. enferm. UERJ [Internet], 2015 [cited 2018 aug 23]; 23(2): 164-71. DOI: http://dx.doi.org/10.12957/reuerj.2015.12540.

5. Moreira NJMP, Souza NVDO, Progianti JM. Work conditions in the hospital: perceptions of obstetric nurses. Rev. enferm. UERJ [Internet], 2017 [cited 2018 aug 23]; 25: e26999. DOI: http://dx.doi.org/10.12957/reuerj.2017.26999.

6. Souza NVDO, Gonçalves FGA, Pires AS, David HMSL. Neoliberalist influences on nursing hospital work process and organization. Rev bras enferm [Internet], 2017 [cited 2018 aug 23]; 70(5): 912-9. DOI: http://dx.doi.org/10.1590/0034-7167-2016-0092.

7. Progianti JM, Moreira NJMP, Prata JA, Vieira MLC, Almeida TA, Vargens OMC. Job insecurity among obstetric nurses. Rev. enferm. UERJ [Internet], 2018 [cited 2019 aug 10]; 26: e33846. DOI: http://dx.doi.org/10.12957/reuerj.2018.33846.

8. Melo CMM, Carvalho CA, Silva LA, Leal JAL, Santos TA, Santos HS. Nurse workforce in state services with direct management: Revealing precarization. Esc. Anna Nery [Internet], 2016 [cited 2017 nov 23]; 20(3): e20160067. Available from: http://www.scielo.br/scielo.php?pid=S1414-81452016000300211\&script=sci_arttext\&tlng=en 
9. Pereira AV. Nurses' daily life: gender relations from the time spent in hospital. Rev. Latino-Am. Enfermagem [Internet], 2015 [cited 2017 nov 23]; 23(5): 945-53. DOI: http://dx.doi.org/10.1590/0104-1169.0485.2635.

10. Ramos TK, Nietsche EA, Cogo SB, Cassenote LG, Martins ABFS. Supervised curricular internship and training of the nurse: developed activities. Rev Enferm UFSM [Internet], 2018 jan-mar [cited 2018 aug 10]; 8(1): 59-71. DOI: http://dx.doi.org/10.5902/2179769228124.

11. Esteves LSF, Cunha ICKO, Negri EC. Supervised internship in undergraduate education in nursing: integrative review. Rev Bras Enferm [Internet], 2018 [cited 2018 aug 10]; 71(suppl 4): 1842-53. DOI: http://dx.doi.org/10.1590/0034-7167-2017-0340.

12. Dejours C. Subjectivity, work and action. Prod. [Internet], 2004 set-dez [cited 2018 jul 16]; 14 (3): 27-34. DOI: http://dx.doi.org/10.1590/S0103-65132004000300004.

13. Dejours C. Addendum: da psicopatologia à psicodinâmica do trabalho. In: Lancman S, Sznelwar L, organizadores. Christophe Dejours: da psicopatologia à psicodinâmica do trabalho. 2a ed. Rio de Janeiro (RJ): Fiocruz; 2008. p. 57-123.

14. Dejours $C$, Abdoucheli $E$, Jayet C. Psicodinâmica do trabalho: contribuições da escola dejouriana à análise da relação prazer, sofrimento e trabalho. São Paulo (SP): Atlas; 2015.

15. Mendes AM. (Org) Psicodinâmica do trabalho: teoria, método e pesquisas. São Paulo (SP): Casa do Psicólogo; 2007.

16. Vinuto JA. Amostragem em bola de neve na pesquisa qualitativa: um debate em aberto. Temáticas [Internet], 2014 ago-dez [cited 2017 sep 8]; 22(44): 203-20. Available from: https://www.ifch.unicamp.br/ojs/index.php/tematicas/article/view/2144/1637.

17. Minayo MCS. O desafio do conhecimento: pesquisa qualitativa em saúde. 14a ed. São Paulo (SP): Hucitec; 2014.

18. Bardin L. Análise de conteúdo. Tradução de Luís Antero Reto e Augusto Pinheiro. 3a ed. São Paulo (SP): Edições 70; 2016.

19. Conselho Nacional de Saúde (Br). Resolução no 466, de 13 de junho de 2012. Diretrizes e normas regulamentadoras de pesquisa envolvendo seres humanos. Brasília (DF): CNS; 2012. [cited 2017 sep 8]. Available from: https://conselho.saude.gov.br/resolucoes/2012/Reso466.pdf.

20. Missio L, Ganassin FMH, Spessoto MMRL, Gomes PLA. Supervised curricular internship: experiences in the nursing graduation Laplage em Revista (Sorocaba) [Internet], 2019 jan-abr [cited 2018 aug 10]; 5(1): 58-70. DOI: https://doi.org/10.24115/S24466220201951611p.58-70.

21. Bordignon SS, Lunardi VL, Barlem EL, Silveira RS, Ramos FR, Dalmolin GL, Barlem JGT. Nursing students facing moral distress: strategies of resistance. Rev. Bras. Enferm. [Internet], 2018 [cited 2019 aug 23]; 71(suppl 4): 1760-7. DOI: http://dx.doi.org/10.1590/0034-7167-2017-0072

22. Wojtowicz B, Hagen B, Daalen-Smith CV. No place to turn: nursing students' experiences of moral distress in mental health settings. Int J Ment Health Nurs [Internet], 2014 [cited 2019 aug 23]; 23(3): 257-64. DOI: http://dx.doi.org/10.1111/inm.12043.

23. Rees CE, Monrouxe LV, Mcdonald LA. 'My mentor kicked a dying woman' sbed...' Analysing UK nursing students' 'mostmemorable' professionalism dilemmas. J Adv Nurs [Internet], 2015 [cited 2019 aug 23]; 71(1): 169-80. DOI: http://dx.doi.org/10.1111/jan.12457.

24. Tavares e Souza MM, Passos JP, Tavares CMM. Suffering and precariousness at work in nursing. J. res. fundam. care. [Internet] 2015 jan-mar [cited 2018 jul 18]; 7(1): 2072-82. DOI: http://dx.doi.org/10.9789/2175-5361.2015.v7i1.2072-2082.

25. Leal JAL, Melo CMM. The nurses' work process in different countries: an integrative review. Rev Bras Enferm [Internet], 2018 [cited 2019 oct 23]; 71(2): 413-23. DOI: http://dx.doi.org/10.1590/0034-7167-2016-0468.

26. Amorim LKA, Souza NVDO, Pires AS, Ferreira ES, Souza MB, Vonk ACRP. The nurse's role: recognition and professional appreciation in the user's view. Rev enferm UFPE on line [Internet], 2017 mai [cited 2019 oct 10]; 11(5): 1918-25. Availabel from: https://periodicos.ufpe.br/revistas/revistaenfermagem/article/download/23341/18945.

27. Jardim DMB, Modena CM. Obstetric violence in the daily routine of care and its characteristics. Rev. Latino-Am. Enfermagem [Internet], 2018 [cited 2019 aug 23];26:e 3069. DOI: http://dx.doi.org/10.1590/1518-8345.2450.3069.

28. Soto-Toussaint LH. Violencia obstétrica. Revista Mexicana de Anestesiología [Internet], 2016 abr-jun [cited 2019 aug 23]; 39(suppl.1): 55-60. Available from: https://www.medigraphic.com/pdfs/rma/cma-2016/cmas161s.pdf

29. Tesser CD, Knobel R, De Aguiar AHF, Diniz SG. Obstetric violence and quaternary prevention: what it is and what to do. Revista Brasileira de Medicina de Família e Comunidade [Internet], 2015 [cited 2018 aug 10]; 10(35): 1-12. DOI: https://doi.org/10.5712/rbmfc10(35)1013.

30. Organização Mundial da Saúde. Prevenção e eliminação de abusos, desrespeito e maus-tratos durante o parto em instituições de saúde. Declaração/ 2014. [cited 2018 dec 15] Available from: http://apps.who.int/iris/bitstream/10665/134588/3/WHO_RHR_14.23_por.pdf.

31. Leal MC, Gama SGN. Inquérito nacional sobre parto e nascimento: Nascer no Brasil. Sumário Executivo Temático da Pesquisa. 2014. [cited $2018 \mathrm{dec} 15]$ Available from: http://www6.ensp.fiocruz.br/nascerbrasil/wpcontent/uploads/2014/11/sumario_executivo_nascer_no_brasil.pdf.

32. Costa RLM, Costa ILS. A point of resistance: nursing, medicine and gender in the hospital context. Caderno Espaço Feminino [Internet], 2017 jul-dez [cited 2018 aug 10]; 30 (2): 357- 66. DOI: https://doi.org/10.14393/CEF-v30n2-2017-20.

33. Dejours C. O fator humano. Tradução de Maria Irene Stocco Bestiol e Maria José Tonelli. Rio de Janeiro (RJ): Fundação Getúlio Vargas, 1997.

34. Dejours C. A banalização da injustiça social. Rio de Janeiro (RJ): Fundação Getúlio Vargas, 2007.

35. Mendes AM, Muller TC. Prazer no trabalho. In: Vieira FO, organizador. Dicionário crítico de gestão e psicodinâmica do trabalho. Curitiba (PR): Juru; 2013. p. 289-92. 